
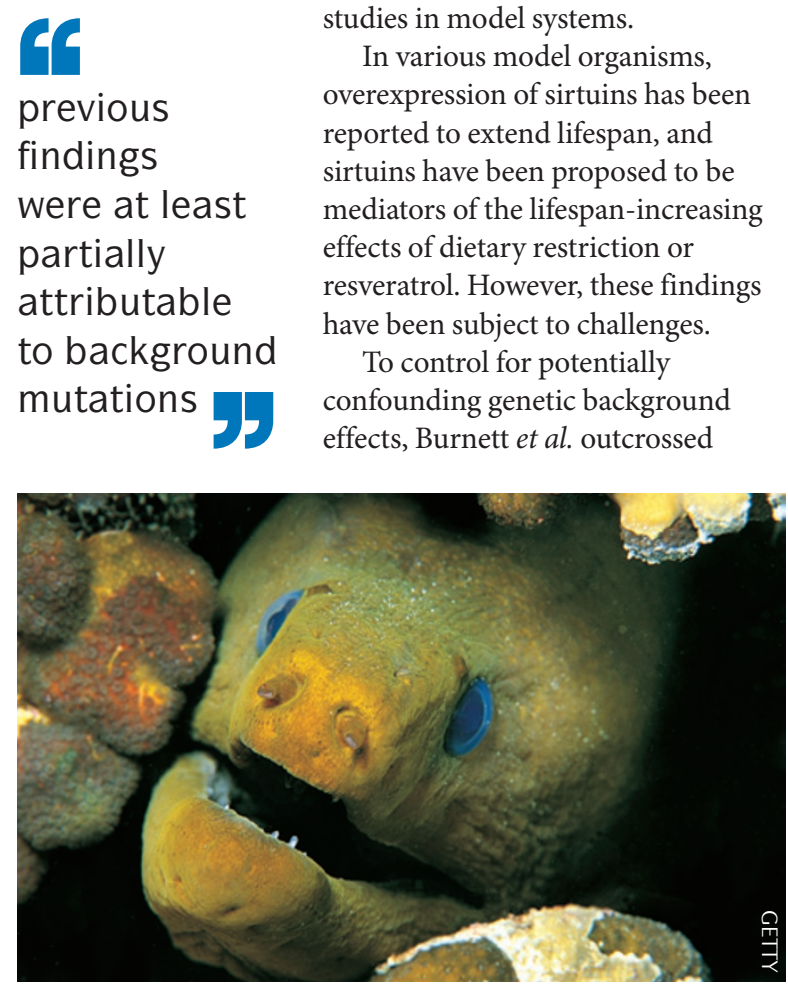

MODEL ORGANISMS

\title{
The dangers lurking in the genetic background
}

Two new reports reveal that the effects of sirtuins on longevity which have aroused great interest in lifespan research - were confounded by background mutations in previous studies. These new studies have important general lessons for genetic studies in model systems.

In various model organisms, overexpression of sirtuins has been reported to extend lifespan, and sirtuins have been proposed to be mediators of the lifespan-increasing effects of dietary restriction or resveratrol. However, these findings have been subject to challenges.

To control for potentially confounding genetic background effects, Burnett et al. outcrossed sir-2.1-overexpressing Caenorhabditis elegans mutants. This eliminated the effect of sir-2.1 on lifespan, despite it still being overexpressed. Instead, the authors isolated the effect on lifespan to a previously unrecognized mutation in the original sir-2.1-overexpressing strain that was removed during outcrossing.

Viswanathan and Guarente performed similar outcrossing experiments, reporting that, although a cleaner genetic background diminished the longevity effect of sir-2.1, the effect was still present. They revised their laboratory's previous estimate of the longevity increase over wild-type strains from $15-50 \%$ to $10-14 \%$.

Burnett et al. also investigated genetic backgrounds in Drosophila melanogaster, in which overexpression of the orthologous gene, Sir2, has typically been achieved by the GAL4-upstream activating sequence (UAS) system. Here, lines that constitutively express the yeast GAL4 transcription factor are used to drive GAL4-dependent Sir2 expression. The authors showed that the lifespan increases that were observed relative to wild-type strains were fully explained by the constitutively expressed GAL4 construct alone. They also showed that resveratrol does not activate D. melanogaster SIR2.

Although the debate regarding the existence of lifespan effect of sirtuins will continue, it is clear that previous findings were at least partially attributable to background mutations. However, these latest results do not condemn sirtuins as unimportant. Aside from their well-supported metabolic roles, Burnett et al. confirmed that sir-2.1 in outcrossed strains still had neuroprotective effects, as previously reported.

These studies serve as a reminder that when comparing results, either within or between experiments, it is crucial to consider the genetic background. Although this research focused on model organisms, it is worth noting that extensive deviation from a wild-type genome occurs in most mammalian cell lines: systems in which this problem could be particularly acute.

Darren J. Burgess

ORIGINAL RESEARCH PAPERS Burnett, C. et al. Absence of effects of Sir2 overexpression on lifespan in C. elegans and Drosophila. Nature 477, 482-485 (2011) | Viswanathan, M. \& Guarente, L. Regulation of Caenorhabditis elegans lifespan by sir-2.1 transgenes. Nature 477, E1-E2 (2011) 